\title{
Neuroimaging of Cerebral Ischemia and Infarction
}

\author{
Carlos Leiva-Salinas, ${ }^{1}$ Max Wintermark, ${ }^{1}$ and Chelsea S. Kidwell ${ }^{2}$ \\ ${ }^{I}$ Department of Radiology, Neuroradiology Division, University of Virginia, Charlottesville, Virginia; ${ }^{2}$ Department of Neurology, \\ Georgetown University Medical Center, 4000 Reservoir Road, NW, Building D, Suite 150, Washington, DC 20007
}

\begin{abstract}
Summary: The imaging workup for patients with suspected acute ischemic stroke has advanced significantly over the past few years. Evaluation is no longer limited to noncontrast computed tomography, but now frequently also includes vascular and perfusion imaging. Although acute stroke imaging has made significant progress in the last few decades with the development of multimodal approaches, there are still
\end{abstract}

many unanswered questions regarding their appropriate use in the setting of daily patient care. It is important for all physicians taking care of stroke patients to be familiar with current multimodal computed tomography and magnetic resonance imaging techniques, including their strengths, limitations, and their role in guiding therapy. Key Words: Stroke, brain infarction, CT, MRI, perfusion, reperfusion therapies.

\section{INTRODUCTION}

In the last few years, substantial advances have been made in the treatment of acute cerebral ischemia. In particular, thrombolytic agents have provided a means to improve the clinical outcome of acute ischemic stroke patients and to reduce the proportion of patients with disability and death. Other promising reperfusion therapies, including mechanical embolectomy, are currently under investigation. With the development of physiologic imaging modalities, such as perfusioncomputed tomography (PCT) and perfusion-weighted magnetic resonance imaging (PWI), and with the advent of fast and reliable noninvasive angiographic techniques, a completely new neuroimaging perspective of ischemic stroke has been introduced. Advanced imaging plays a growing role not only in the diagnosis of stroke but also in the selection of patients for acute therapies. This review will briefly discuss the individual components of multimodal magnetic resonance (MR) and multimodal CT imaging, review the role of these imaging modalities in the characterization of ischemic penumbra and in the selection of patients for reperfusion therapies and, finally, discuss the future steps needed in this field.

Electronic supplementary material The online version of this article (doi:10.1007/s13311-010-0004-2) contains supplementary material, which is available to authorized users.

Address correspondence and reprint requests to: Chelsea S. Kidwell, M.D., Department of Neurology, Georgetown University Medical Center, 4000 Reservoir Road, NW, Building D, Suite 150, Washington, DC 20007. E-mail: ck256@georgetown.edu.

\section{FROM STRUCTURAL TO FUNCTIONAL MULTIMODAL STROKE IMAGING}

In the past, acute stroke imaging consisted of simple structural imaging modalities. As such, noncontrast CT (NCT) was typically used to rule out hemorrhage and other stroke mimics and to potentially detect the presence of early, subtle acute ischemic signs. With the development of multislice CT, new MR sequences, and fast and reliable post-processing software, imaging has now the capability to evaluate acute stroke as a dynamic and evolving process - in contrast to the previous static all-or-none approach. In particular, the neuroimaging community has made important advances in operationally defining ischemic "penumbra" with $\mathrm{CT}$ and MRI techniques currently available in the acute stroke setting.

\section{Penumbral imaging}

Prior to the introduction of an effective therapy capable of limiting the size of an acute cerebral infarct, the ischemic penumbra was more a theoretical rather than a practical matter. Following an arterial occlusion, a core of brain tissue dies rapidly. Surrounding this infarct core is an area of brain that is hypoperfused, but still viable. This area at risk, but comprising potentially salvageable tissue, is called the ischemic penumbra [1-3]. The evolution of the infarct core and penumbra is a dynamic process that depends mainly on the collateral flow, but also on the timing and extent of reperfusion of the ischemic tissue. If the vascular occlusion remains, the infarct core will likely grow and progressively replace 
the penumbra. If prompt recanalization is achieved, either spontaneous or thrombolysis related, the penumbra may be rescued from infarction [4].

With the approval of intravenous recombinant tissue plasminogen activator (rt-PA) for the treatment of acute ischemic stroke, the concept of the penumbra has gained increasing importance as an indicator of the volume of brain tissue that is potentially salvageable after a successful treatment [5]. Indeed, at the present time, intravenous rt-PA is routinely accepted as the only proven treatment for acute ischemic stroke in the first 3-4.5 h after symptom onset [6]. However, very few patients are admitted in this narrow time window and, as a result, less than $10 \%$ of acute ischemic stroke patients are treated [7]. It has been hypothesized, however, that intravenous rt-PA or other reperfusion therapies could be safely administered in an extended time window in selected patients with a sufficient amount of salvageable penumbra $[8,9]$, which would allow a much larger percentage of stroke patients to be safely treated. This concept emphasizes the urgent need for advanced imaging techniques capable of delineating the ischemic penumbra in the acute stroke setting [10]. During the last decade, several operational definitions of ischemic penumbra have been proposed using MRI and, increasingly, CT. Furthermore, some studies have suggested a favorable clinical outcome with thrombolytic therapies administered to patients selected on the basis of penumbral imaging in an extended time window [11].

\section{Vascular imaging}

Vascular imaging, combined with penumbral imaging, provides another fundamental piece of information in the workup of acute ischemic stroke patients and in the assessment of the response to reperfusion therapies. Patients should be differentiated based on the amount of ischemic penumbra as well as on the initial angiographic findings since the location and the extent of vascular obstruction have important prognostic value in predicting response to thrombolytics [12, 13]. For instance, patients with a "carotid- $\mathrm{T}$ " occlusion, proximal middle cerebral artery (MCA) occlusion, tandem lesions or significant thrombus burden might respond poorly to intravenous thrombolytics, and therefore may be appropriate candidates for subsequent intra-arterial or mechanical thrombolysis [14-16].

\section{Multimodal stroke imaging}

By combining parenchymal, penumbral, and vascular imaging in one single study, multimodal $\mathrm{CT}$ and MR provide a comprehensive hyperacute stroke imaging evaluation. Both approaches can 1) exclude intracranial hemorrhage (ICH) and other mimics of ischemic stroke, 2) provide reliable information about the location and extent of ischemia, 3) identify the existence and extent of potentially salvageable brain, and 4) identify the site of vascular occlusion and degree of collateral flow. Multimodal CT and MRI have been shown to be fast, reliable, and effective in improving stroke detection and characterization in the emergency setting [17], and to have the potential to select patients that may benefit from reperfusion therapy [11].

\section{ACUTE STROKE MRI PROTOCOL}

A typical multimodal stroke MRI protocol consists of T2/fluid-attenuated inversion recovery (FLAIR)-, T2*-, diffusion (DWI)- and perfusion (PWI)-weighted imaging and MR angiography (MRA) [17]. This protocol can be performed in $<15-30 \mathrm{~min}$.

\section{Location and extent of the acute ischemia: DWI}

DWI provides an image signal that is dependent on the molecular motion of water [18]. Cerebral ischemia leads to a disruption of energy metabolism, with disruption with failure of the $\mathrm{Na}+/ \mathrm{K}+$ ionic pump causing cytotoxic edema [1]. Intracellular water flow leads to a reduced extracellular volume, whereby water mobility is relatively facilitated and, therefore, to a net reduction of water diffusion [4, 18]. This phenomenon can be detected as a hyperintense signal on DWI scans within minutes of vessel occlusion (FIG. 1) [4, 18]. In addition, an apparent diffusion coefficient map can be constructed to quantify the extent of restricted diffusion.

The main advantage of MRI over CT is that DWI is the most sensitive method to date for the depiction of ischemia in the hyperacute stage $[19,20]$. It should be noted, however, that DWI lesions can be at least partially reversible in the very early phase of ischemia and, as such, the initial DWI abnormality does not necessarily reflect the infarct core [21]. An additional advantage of DWI is its ability to distinguish acute from chronic ischemia, thereby allowing new lesions to be identified in patients even when these are near or within areas of prior ischemic injury. DWI can also visualize small lesions not evident on CT scans, particularly in the posterior fossa; the location and pattern of these lesions often provide insight into the underlying stroke mechanism.

\section{Exclusion of other stroke mimics and study of the brain parenchyma: T2-/FLAIR images}

Cerebral ischemia usually becomes visible within the first 3-8 h after stroke onset on T2-weighted and FLAIR images [22-24]. A recent study has shown that, in acute stroke patients with a DWI-positive lesion, a normal FLAIR image suggests a time window of less than $3 \mathrm{~h}$ with $>90 \%$ specificity and positive predictive value. 


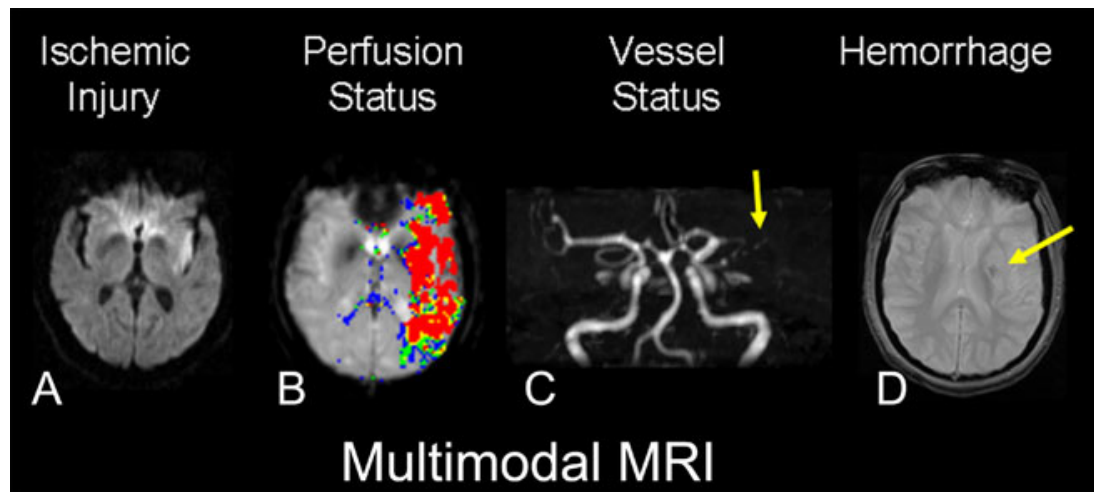

FIG. 1. Example of multimodal MRI in acute stroke: (A) DWI showing hyperintense ischemic region in the left insula. (B) Tmax perfusion MRI demonstrating left MCA perfusion deficit. (C) Intracranial MRA demonstrating left MCA occlusion (arrow). (D) gradient echo MRI emonstrating small region of hemorrhagic transformation (arrow). $\mathrm{MRI}=$ Magnetic resonance imaging; $\mathrm{DWI}=$ diffusion-weighted $\mathrm{MR} ; \mathrm{MCA}=$ middle cerebral artery. With kind permission from Kidwell and Wintermark [62, figure 1, page 22]. Copyright, Springer Science and Business Media. (High resolution version of this image is available in the electronic supplementary material.)

Thereby, a mismatch between DWI-positive and DWInegative FLAIR images may be a reliable approach for the identification of unknown onset stroke patients who are likely to benefit from thrombolysis [23]. FLAIR images are also highly sensitive in detecting other lesions, including subarachnoid hemorrhage and acute cerebral venous sinus thrombosis [25-27].

\section{Exclusion of intracranial hemorrhage: $\mathrm{T} 2 *$-weighted images}

Although CT is the standard method used to rule out $\mathrm{ICH}$, gradient-recalled echo and other $\mathrm{T} 2 *$ sequences are equally - if not more - sensitive for the detection of acute ICH, even by relatively inexperienced readers (FIG. 1) [28, 29].

Furthermore, small hemosiderin deposits not apparent on CT scans can be detected by $\mathrm{T} 2 *$-weighted images [29]. These chronic microbleeds are an indicator of a hemorrhage-prone vasculopathy and are associated with an increased risk of spontaneous ICH. The relationship between chronic microbleeds and post-thrombolysisrelated hemorrhage is still being debated [20, 21]. In the BRASIL study [30], the odds ratio for symptomatic hemorrhage following the intravenous administration of rt-PA in patients with microbleeds versus without was 2.23 (95\% confidence interval, 0.67-6.97). The authors concluded that any increased risk of thrombolytic therapy in the setting of microbleeds is likely to be small and unlikely to exceed the benefits of treatment. Due to the very small number of patients with multiple microbleeds, no reliable conclusions could be drawn regarding the risk in this subgroup.

Finally, due to the high concentration of deoxyhemoglobin in the acute thrombus, $\mathrm{T} 2 *$-weighted images are able to detect intraluminal clots as linear or dotshaped low-signal areas of magnetic susceptibility [24].

\section{Location and extent of the arterial occlusion: MR angiography (MRA)}

3-Dimensional time-of-flight MRA is the standard technique for the examination of intracranial vessels. This sequence depicts vascular flow by repeatedly applying saturation pulses to a volume of tissue. Stationary protons in the excited plane become saturated by the repeated pulses, whereas inflowing blood protons are not and, therefore, produce a relatively increased signal intensity [24, 31].

Contrast enhanced-MRA is the technique of choice for the study of the extracranial arteries. It relies on the injection of gadolinium to reduce the $\mathrm{T} 1$ relaxation time of tissue and to generate contrast between the intravascular lumen and surrounding tissues [32]. Unlike time-of-flight MRA, vascular contrast is relatively independent of flow dynamics and, therefore, artifacts are substantially reduced.

MRA is employed for the detection of the location of the vascular occlusion and/or stenosis in both the intracranial and extracranial vasculature (FIG. 1) [24, 32].

\section{Location and extent of the hypoperfused area: PWI}

PWI provides a measurement of cerebral perfusion by tracking the bolus passage of a paramagnetic MRI contrast agent administered intravascularly [33]. Using a dynamic contrast-enhanced technique, the temporal passage of gadolinium is tracked in repeated contiguous slices throughout all of the brain volume using $\mathrm{T} 2 *$. The tissue signal change caused by the susceptibility effect of gadolinium is used to create a hemodynamic time-tosignal intensity curve and then to generate a set of semiquantitative perfusion maps [34]. These include maps of relative cerebral blood flow (CBF), mean transit time (MTT), cerebral blood volume (CBV), and time-topeak (TTP; including Tmax, which is the TTP of the residue function) measures. CBV reflects the blood volume per unit of brain, MTT designates the average 
time required by the contrast bolus to cross the capillary network, CBF relates to the volume of blood flowing per brain mass during a time interval of $1 \mathrm{~min}$, and TTP and Tmax measure the time to peak of the contrast agent within the vessel. The relationship between $\mathrm{CBF}$ and $\mathrm{CBV}$ is expressed by the equation $\mathrm{CBF}=\mathrm{CBV} / \mathrm{MTT}[35$, 36].

The main advantage of MR PWI over PCT is the whole brain perfusion coverage of the former that allows the detection of small but potentially clinically relevant hypoperfusion areas. The main disadvantage of PWI is that the measures are semiquantiative rather than absolute.

\section{ACUTE STROKE CT PROTOCOL}

\section{Multimodal CT stroke protocol}

A typical multimodal stroke CT protocol consists of NCT, PCT, and CT angiography (CTA). This protocol can be performed in $<10$ min.

\section{Exclusion of other stroke mimics and assessment of early CT signs of ischemia: NCT}

Due to its wide availability, speed, and patient tolerance, NCT has traditionally been the first-line imaging modality for the evaluation of acute ischemic stroke. NCT is mainly used to rule-out intracranial hemorrhage.

In some cases, NCT can identify signs of early ischemia in hyperacute stroke [37-39]. These include the insular ribbon sign, obscuration of the lentiform nucleus, and the presence of hyperdense vessels (FIGS. 2

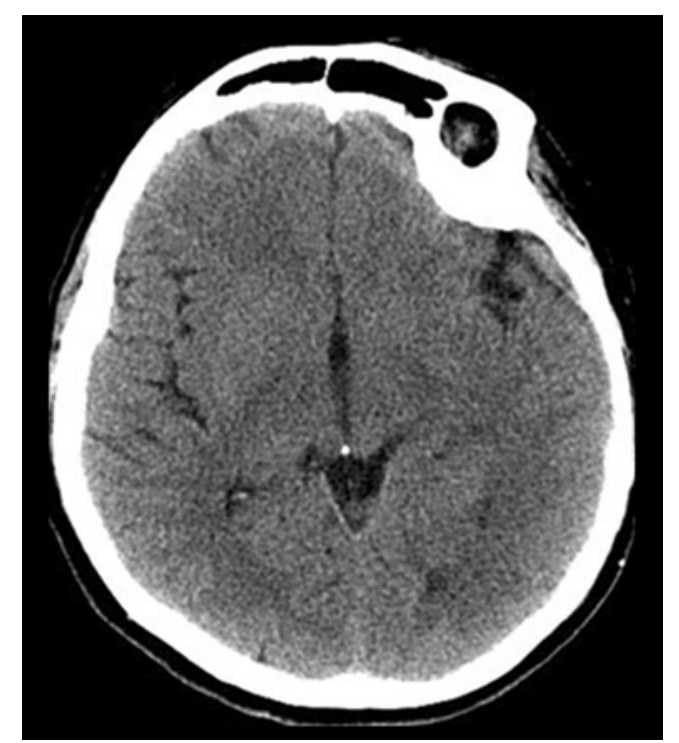

FIG. 2. NCT scan of the brain in a 71-year-old male obtained $4 \mathrm{~h}$ after the onset of symptoms shows an obscuration of the left lentiform nucleus. NCT = Noncontrast computed tomography. (High resolution version of this image is available in the electronic supplementary material.) and 3). The first two signs allude to the partial disappearance or loss of definition of the gray-white matter interface. The latter, caused by the presence of an acute hyperdense thrombus in a vessel (often the middle cerebral artery), 1does not represent a sign of infarction but, rather, is a hallmark of an acute thrombo-embolic event. Although those early signs can be helpful in confirming the location and diagnosis of ischemia, their detection is difficult and depends on the experience of the reader. Hence, infarct detection with NCT in the first $3 \mathrm{~h}$ after onset is a challenge, with sensitivity values as low as $25 \%$ in comparison with DWI-MR [40]. It should be noted that the relationship between early ischemic changes visible on the CT images and adverse outcomes after rt-PA treatment is not straightforward and that the presence of early infarct signs on the NCT scan should not be considered a contraindication to thrombolytic treatment [41, 42].

Unlike these subtle hyperacute ischemic changes, obvious hypoattenuation is highly specific for tissue infarct, [43] and its extent is predictive of the risk of hemorrhagic transformation [44], clinical outcome, and final infarct volume. Frank hypoattenuation on the CT scan, particularly when it involves more than one-third of the MCA territory, has been used in most clinical trials as an exclusion criterion for thrombolysis due to the potential increased risk for hemorrhagic transformation [45].

Despite its advantages, NCT provides solely structuraland not physiologic - information, and it cannot reliably differentiate between irreversibly damaged brain tissue and penumbral tissue.

\section{Location and extent of the arterial occlusion: CTA}

CTA allows a fast and detailed evaluation of the intraand extracranial vasculature in one single contrast injection $[46,47]$. Its speed and high spatial resolution make it a powerful tool by which to evaluate the vasculature in acute stroke patients and support its inclusion in an acute stroke imaging protocol $[46,47]$. The information obtained from the arterial imaging may be used to guide therapy. Several studies have shown an association between occlusion site, recanalization success rate, and clinical outcome [14-16]. Currently available multidetector CT scanners may have the ability to dynamically assess the collateral flow [48]. This additional piece of information might play a role in the therapeutic workup.

Location and extent of the hypoperfused area: PCT

PCT imaging relies on the speed of modern helical CT scanners that can sequentially trace the entry and washout of a bolus of standard iodinated contrast agent injected into a peripheral vein [36]. Analysis of the signal density during the passage of the contrast agent provides information on brain capillary perfusion [35]. The linear 

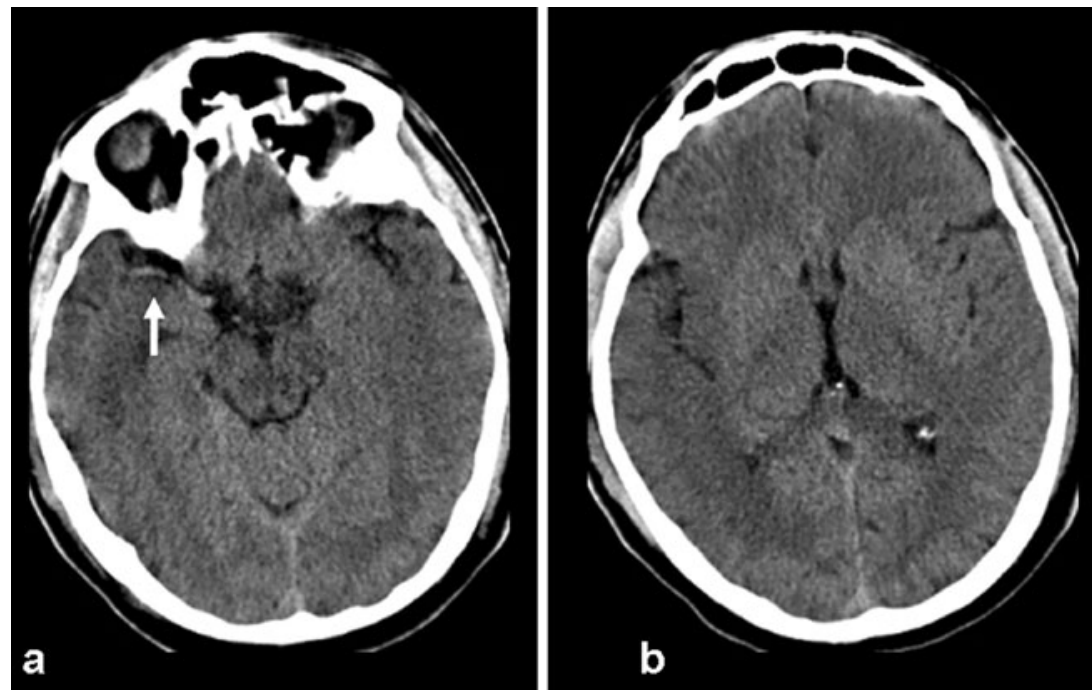

FIG. 3. NCT scan of the brain in a 66-year-old male obtained $5 \mathrm{~h}$ after the onset of symptoms shows hyperdense right MCA (a) (arrow) and insular cortical ribbon loss signs (b). (High resolution version of this image is available in the electronic supplementary material.)

relationship between the concentration of the contrast agent and CT tissue density provides quantitative assessments of cerebral perfusion, which is a significant advantage of PCT over PWI. As with MRI, PCT evaluation of brain perfusion consists of 3 main sets of values and parametric maps: CBV, MTT, CBF (FIG. 4).

The main limitation of PCT is its inability to image the whole brain, as conventional scanners are limited to a 2to 4-cm section of brain tissue per contrast bolus. Progressive introduction of the new volumetric CT scanners, offering whole brain coverage, is likely to overcome this limitation in the near future [48].

Of note, a recent study has demonstrated that not only does PCT provide an assessment of hemodynamic status, but that this assessment in turn improves diagnostic accuracy for ischemia over that achieved with NCT alone or with combined NCT and CTA, even when read by inexperienced radiologists [17].

\section{NEUROIMAGING FOR THE DIFFERENTIATION OF INFARCT CORE AND PENUMBRA}

Differentiation of the infarct core and ischemic penumbra relies on the concept of cerebral vascular autoregulation $[35,49,50]$. Vascular autoregulation consists of complex neurobiochemical mechanisms that guarantee the stability of regional CBF despite changes in the local neuronal metabolic activity or in the local arterial perfusion pressure. It notably allows for a preand postcapillary dilatation in response to a decreased perfusion pressure in order to maintain a constant CBF. In the penumbra, where autoregulation is intact or mildly jeopardized, MTT is prolonged; however, the CBV is maintained or increased due to this compensatory vasodilatation. In tissue proceeding to infarction, $\mathrm{CBF}$ is low and autoregulation is impaired, leading to low CBV values with a loss of ability to maintain vasodilatory compensation [35, 49].

\section{MRI}

Initial MRI approaches to characterizing the ischemic core and penumbra were based on the simplified assumption that the DWI abnormality reflects the irreversibly damaged infarct core, whereas the PWI abnormality reflects the overall area of hypoperfusion [51]. The volumetric difference between these images, i.e, the PWI/DWI-mismatch, was thought to represent the ischemic penumbra (FIG. 1). Since these initial studies, however, it has been shown that this model does not take into consideration that DWI lesions do not necessarily turn into infarction and that the PWI abnormalities may also represent areas of benign oligemia that are not at risk $[21,52]$. Moreover, to date, threshold approaches to differentiating core and penumbra have also not been shown to have high levels of accuracy. A study of 10 different MR perfusion methods using different postprocessing revealed differences in hypoperfused tissue volumes depending on the parameter used to calculate them [53]. In current clinical practice, however, Tmax and MTT are the parameters most often used. Efforts are underway to identify more accurate multivariate MRI models that incorporate information on a voxel level from both perfusion and apparent diffusion coefficient maps. These approaches have the potential to have greater sensitivity and specificity in predicting the tissue core and penumbra in the hyperacute window [54]. 


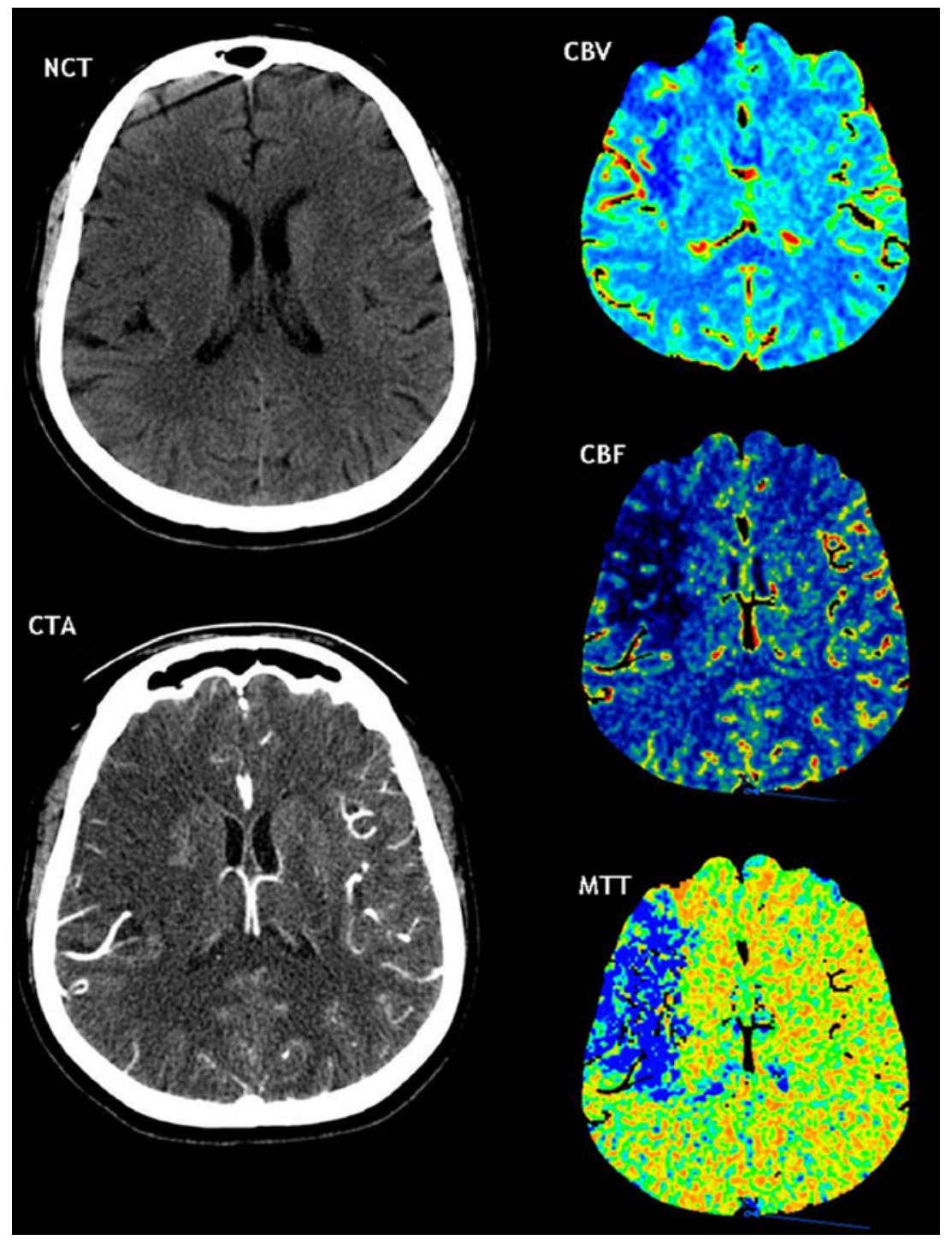

FIG. 4. Multimodal CT imaging in a 63-year-old female patient with acute stroke obtained $4 \mathrm{~h}$ after symptom onset. NCT scan shows subtle loss of the gray-white matter interface in the right parietal lobe. CTA demonstrates a paucity of MCA distal branches in the affected brain area; no proximal MCA occlusion was found. PCT parametric maps show a nearly complete match between the MTT and CBV parametric maps. The patient was not treated with rt-PA. CTA = CT angiography; PCT = perfusion-CT; MTT = mean transit time; $\mathrm{CBV}=$ cerebral blood volume; rt-PA = recombinant tissue plasminogen activator. (High resolution version of this image is available in the electronic supplementary material.)

\section{PCT}

By combining quantitative MTT and CBV results, PCT has the capacity to reliably identify the reversible ischemic penumbra and the irreversible infarct core in acute stroke patients [55]. In the infarct core, MTT is prolonged and CBV is low, whereas in the penumbra, MTT is prolonged but CBV is high due to vasodilatory compensation. Quantitatively, the parameter that most accurately describes the tissue at risk of infarction is the relative MTT, with an optimal threshold of 145\% [55]. The parameter that most accurately describes the infarct core on admission is the absolute CBV, with an optimal threshold of $2.0 \mathrm{ml} / 100 \mathrm{~g}$ [54]. The PCT correlate for the infarct core and ischemic penumbra has been validated by prospective studies and been shown to compare to the PWI/DWI MR mismatch $[50,56]$.

\section{NEUROIMAGING FOR THE SELECTION OF PATIENTS FOR THROMBOLYTIC THERAPY: CURRENT EVIDENCE AND FUTURE PERSPECTIVES}

There is a growing body of data suggesting that multimodal imaging can be used for selecting patients for acute reperfusion therapies. The DIAS and DEDAS trials randomized patients within a 3- to 9-h time window after stroke setting and with a DWI/PWI mismatch of at least $20 \%$ either to placebo or to escalating doses of a novel thrombolytic drug, desmoteplase $[8,9]$. Those patients who received a placebo or an ineffective dosage showed a lower recanalization rate and an unfavorable outcome. In comparison, those patients who achieved early vessel 
recanalization and reperfusion of penumbral tissue, showed a significant clinical benefit, with $60 \%$ of the patients from the most effective dose tier having an excellent clinical outcome [8].

In the DIAS 2 study [57], patients were enrolled based on a mismatch diagnosed either by MR or by PCT. Unfortunately, this study did not show any benefit in clinical outcome in either of the treatment groups, contrasting sharply with the previous findings with desmoteplase in the DIAS and DEDAS trials.

The DEFUSE study showed that baseline MRI findings can be used to identify groups of patients who are more likely to benefit from thrombolytic therapy and, potentially, other forms of reperfusion therapy [11]. Patients with a baseline mismatch between PWI and DWI of at least $20 \%$ and a reduction in abnormal perfusion volume of at least $10 \mathrm{~mL}$ had a better clinical outcome. Data from this study suggested that a mismatch ratio (PWI volume - DWI volume/DWI volume) of 2.6 provided the highest sensitivity and specificity for identifying patients in whom reperfusion was associated with a favorable response. However, no benefit could be expected if early recanalization of the occluded vessel failed, even in the presence of a large mismatch [58].

The most recent data supporting the use of penumbral selection comes from the Echoplanar Imaging Thrombolytic Evaluation Trial (EPITHET), which was a randomized, controlled multicenter trial of IV rt-PA versus placebo 3-6 h from onset [59]. All patients underwent pretreatment MRI, but findings were not used to determine eligibility. While the primary outcome, infarct growth measured by the geometric mean, was not statistically significant, a secondary outcome of growth, namely, the median growth ratio, was significant in favor of attenuated growth in the rt-PA arm in mismatch patients. The investigators also found that reperfusion was significantly correlated with growth attenuation and improved outcomes. Unfortunately, they also found there were insufficient numbers of non-mismatch patients to compare groups.

Although acute stroke imaging has made significant progress in the last few decades with the development of multimodal approaches, there are still many unanswered questions regarding their appropriate use in the daily patient care. The role and utility of multimodal imaging of intravenously administered rt-PA candidates within $4.5 \mathrm{~h}$ is unclear-further clinical trial data are needed to weigh the risks and benefits of this approach. Clinical trials of acute reperfusion therapies in an extended time window have shown mixed results. New trials should overcome the limitations of these previous studies. Two main barriers that need to be solved are the lack of standardization and the validation of analytic approaches, especially for the penumbra imaging [60]. The various trials published to date used different perfusion models, different sequence parameters, and even different thresholds for defining core and penumbra [61]. After the key step of standardization, additional large multicenter trials are needed to validate these techniques to definitely demonstrate that advanced imaging selection for reperfusion therapies improves clinical outcome. Approaches to vascular imaging should also be standardized and this information incorporated into protocols to optimize trial design, including the outcome assessment of both recanalization and reperfusion $[12,61]$.

\section{CONCLUSIONS}

Multimodal MRI and CT techniques provide a comprehensive assessment of the size, location, and severity of cerebral ischemia, hemodynamic compromise, and vessel occlusion. The combined information can be used to determine the presence and extent of infarct core and penumbra. Clinical trials to date suggest that this information may be used to select optimal candidates for reperfusion therapies. However, future studies are needed to provide a valid standardized penumbra model that can be widely employed by the neuroimaging community and to demonstrate that multimodal imaging selection improves outcomes.

Acknowledgments: Dr. Kidwell receives funding from NIHNINDS grant P50 NS044378 and NIH-NINDS U54 NS057405.

\section{REFERENCES}

1. Astrup J, Siesjo BK, Symon L. Thresholds in cerebral ischemia-the ischemic penumbra. Stroke 1981;12:723-725.

2. Hossmann KA. Viability thresholds and the penumbra of focal ischemia. Ann Neurol 1994;36:557-565.

3. Hossmann KA. Neuronal survival and revival during and after cerebral ischemia. Am J Emerg Med 1983;1:191-197.

4. Read SJ, Hirano T, Abbott DF, et al. The fate of hypoxic tissue on $18 \mathrm{~F}$-fluoromisonidazole positron emission tomography after ischemic stroke. Ann Neurol 2000;48:228-235.

5. Tissue plasminogen activator for acute ischemic stroke. the national institute of neurological disorders and stroke rt-PA stroke study group. N Engl J Med 1995;333:1581-1587.

6. Schellinger PD, Warach S. Therapeutic time window of thrombolytic therapy following stroke. Curr Atheroscler Rep 2004;6:288-294.

7. Bambauer KZ, Johnston SC, Bambauer DE, Zivin JA. Reasons why few patients with acute stroke receive tissue plasminogen activator. Arch Neurol 2006;63:661-664.

8. Hacke W, Albers G, Al-Rawi Y, et al. The desmoteplase in acute ischemic stroke trial (DIAS): A phase II MRI-based 9-hour window acute stroke thrombolysis trial with intravenous desmoteplase. Stroke 2005;36:66-73.

9. Furlan AJ, Eyding D, Albers GW, et al. Dose escalation of desmoteplase for acute ischemic stroke (DEDAS): Evidence of safety and efficacy 3 to 9 hours after stroke onset. Stroke 2006;37:1227-1231.

10. Kaste M. Reborn workhorse, CT, pulls the wagon toward thrombolysis beyond 3 hours. Stroke 2004;35:357-359.

11. Albers GW, Thijs VN, Wechsler L, et al. Magnetic resonance imaging profiles predict clinical response to early reperfusion: The 
diffusion and perfusion imaging evaluation for understanding stroke evolution (DEFUSE) study. Ann Neurol 2006;60:508-517.

12. von Kummer R. Treatment of acute stroke: A misconception. Neuroradiology 2010;52:337-339.

13. Tan JC, Dillon WP, Liu S, Adler F, Smith WS, Wintermark M. Systematic comparison of perfusion-CT and CT-angiography in acute stroke patients. Ann Neurol 2007;61:533-543.

14. Zaidat OO, Suarez JI, Santillan C, et al. Response to intra-arterial and combined intravenous and intra-arterial thrombolytic therapy in patients with distal internal carotid artery occlusion. Stroke 2002;33:1821-1826.

15. Rubiera M, Ribo M, Delgado-Mederos R, et al. Tandem internal carotid artery/middle cerebral artery occlusion: An independent predictor of poor outcome after systemic thrombolysis. Stroke 2006;37:2301-2305.

16. Saqqur M, Uchino K, Demchuk AM, et al. Site of arterial occlusion identified by transcranial doppler predicts the response to intravenous thrombolysis for stroke. Stroke 2007;38:948-954.

17. Hopyan J, Ciarallo A, Dowlatshahi D, et al. Certainty of stroke diagnosis: Incremental benefit with CT perfusion over noncontrast CT and CT angiography. Radiology 2010;255:142-153.

18. Srinivasan A, Goyal M, Al Azri F, Lum C. State-of-the-art imaging of acute stroke. Radiographics 2006;26 (suppl 1):S7595.

19. Fiebach JB, Schellinger PD, Jansen O, et al. CT and diffusionweighted MR imaging in randomized order: Diffusion-weighted imaging results in higher accuracy and lower interrater variability in the diagnosis of hyperacute ischemic stroke. Stroke 2002;33:22062210.

20. Saur D, Kucinski T, Grzyska U, et al. Sensitivity and interrater agreement of CT and diffusion-weighted MR imaging in hyperacute stroke. AJNR Am J Neuroradiol 2003;24:878-885.

21. Fiehler J, Knudsen K, Kucinski T, et al. Predictors of apparent diffusion coefficient normalization in stroke patients. Stroke 2004;35:514-519.

22. Mohr JP, Biller J, Hilal SK, et al. Magnetic resonance versus computed tomographic imaging in acute stroke. Stroke 1995;26:807-812.

23. Thomalla G, Rossbach P, Rosenkranz M, et al. Negative fluidattenuated inversion recovery imaging identifies acute ischemic stroke at 3 hours or less. Ann Neurol 2009;65:724-732.

24. Gonzalez RG, Schaefer P. Conventional MRI and MR angiography of stroke. In: Acute ischemic stroke: imaging and intervention. 1st edn. Berlin: Springer, 2006, 115-137.

25. Fiebach JB, Schellinger PD, Geletneky K, et al. MRI in acute subarachnoid haemorrhage; findings with a standardised stroke protocol. Neuroradiology 2004;46:44-48.

26. Boukobza M, Crassard I, Bousser MG, Chabriat H. MR imaging features of isolated cortical vein thrombosis: Diagnosis and followup. AJNR Am J Neuroradiol 2009;30:344-348.

27. Lovblad KO, Bassetti C, Schneider J, et al. Diffusion-weighted MR in cerebral venous thrombosis. Cerebrovasc Dis 2001;11:169176.

28. Fiebach JB, Schellinger PD, Gass A, et al. Stroke magnetic resonance imaging is accurate in hyperacute intracerebral hemorrhage: A multicenter study on the validity of stroke imaging. Stroke 2004;35:502-506.

29. Kidwell CS, Chalela JA, Saver JL, et al. Comparison of MRI and $\mathrm{CT}$ for detection of acute intracerebral hemorrhage. JAMA 2004;292:1823-1830.

30. Fiehler J, Albers GW, Boulanger JM, et al. Bleeding risk analysis in stroke imaging before thromboLysis (BRASIL): Pooled analysis of T2*-weighted magnetic resonance imaging data from 570 patients. Stroke 2007;38:2738-2744.

31. Miyazaki M, Lee VS. Nonenhanced MR angiography. Radiology 2008;248:20-43.

32. Leclerc X, Gauvrit JY, Nicol L, Pruvo JP. Contrast-enhanced MR angiography of the craniocervical vessels: A review. Neuroradiology 1999;41:867-874.

33. Grandin CB. Assessment of brain perfusion with MRI: Methodology and application to acute stroke. Neuroradiology 2003;45:755766.
34. Rosen BR, Belliveau JW, Vevea JM, Brady TJ. Perfusion imaging with NMR contrast agents. Magn Reson Med 1990;14:249-265.

35. Wintermark M, Maeder P, Thiran JP, Schnyder P, Meuli R. Quantitative assessment of regional cerebral blood flows by perfusion CT studies at low injection rates: A critical review of the underlying theoretical models. Eur Radiol 2001;11:12201230 .

36. Eastwood JD, Lev MH, Provenzale JM. Perfusion CT with iodinated contrast material. AJR Am J Roentgenol 2003;180:312.

37. Truwit CL, Barkovich AJ, Gean-Marton A, Hibri N, Norman D. Loss of the insular ribbon: Another early CT sign of acute middle cerebral artery infarction. Radiology 1990;176:801-806.

38. Tomura N, Uemura K, Inugami A, Fujita H, Higano S, Shishido F. Early CT finding in cerebral infarction: Obscuration of the lentiform nucleus. Radiology 1988;168:463-467.

39. Tomsick TA, Brott TG, Chambers AA, et al. Hyperdense middle cerebral artery sign on CT: Efficacy in detecting middle cerebral artery thrombosis. AJNR Am J Neuroradiol 1990;11:473477.

40. Barber PA, Darby DG, Desmond PM, et al. Identification of major ischemic change. diffusion-weighted imaging versus computed tomography. Stroke 1999;30:2059-2065.

41. Patel SC, Levine SR, Tilley BC, et al. Lack of clinical significance of early ischemic changes on computed tomography in acute stroke. JAMA 2001;286:2830-2838.

42. Roberts HC, Dillon WP, Furlan AJ, et al. Computed tomographic findings in patients undergoing intra-arterial thrombolysis for acute ischemic stroke due to middle cerebral artery occlusion: Results from the PROACT II trial. Stroke 2002;33:1557-1565.

43. von Kummer R, Bourquain H, Bastianello S, et al. Early prediction of irreversible brain damage after ischemic stroke at CT. Radiology 2001;219:95-100.

44. Larrue V, von Kummer RR, Muller A, Bluhmki E. Risk factors for severe hemorrhagic transformation in ischemic stroke patients treated with recombinant tissue plasminogen activator: A secondary analysis of the European-Australasian acute stroke study (ECASS II). Stroke 2001;32:438-441.

45. Hacke W, Kaste M, Fieschi C, et al. Intravenous thrombolysis with recombinant tissue plasminogen activator for acute hemispheric stroke. The European Cooperative Acute Stroke Study (ECASS). JAMA 1995;274:1017-1025.

46. Prokop M. Multislice CT angiography. Eur J Radiol 2000;36:8696.

47. Lev MH, Farkas J, Rodriguez VR, et al. CT angiography in the rapid triage of patients with hyperacute stroke to intraarterial thrombolysis: Accuracy in the detection of large vessel thrombus. J Comput Assist Tomogr 2001;25:520-528.

48. Salomon EJ, Barfett J, Willems PW, Geibprasert S, Bacigaluppi S, Krings T. Dynamic CT angiography and CT perfusion employing a 320-detector row CT: Protocol and current clinical applications. Klin Neuroradiol 2009;19:187-196.

49. Wintermark M, Reichhart M, Thiran JP, et al. Prognostic accuracy of cerebral blood flow measurement by perfusion computed tomography, at the time of emergency room admission, in acute stroke patients. Ann Neurol 2002;51:417-432.

50. Heiss WD, Sobesky J, Hesselmann V. Identifying thresholds for penumbra and irreversible tissue damage. Stroke 2004;35:26712674.

51. Jansen O, Schellinger P, Fiebach J, Hacke W, Sartor K. Early recanalisation in acute ischaemic stroke saves tissue at risk defined by MRI. Lancet 1999;353:2036-2037.

52. Kidwell CS, Alger JR, Saver JL. Beyond mismatch: Evolving paradigms in imaging the ischemic penumbra with multimodal magnetic resonance imaging. Stroke 2003;34:2729-2735.

53. Kane I, Carpenter T, Chappell F, et al. Comparison of 10 different magnetic resonance perfusion imaging processing methods in acute ischemic stroke: Effect on lesion size, proportion of patients with diffusion/perfusion mismatch, clinical scores, and radiologic outcomes. Stroke 2007;38:3158-3164.

54. Mechanical Retrieval and Recanalization of Stroke Clots Using Embolectomy (MR RESCUE). Available at: http://clinicaltrials. 
gov/ct2/show/NCT00389467?term=MR+rescue\&rank=1. Accessed June 28, 2010

55. Wintermark M, Flanders AE, Velthuis B, et al. Perfusion-CT assessment of infarct core and penumbra: Receiver operating characteristic curve analysis in 130 patients suspected of acute hemispheric stroke. Stroke 2006;37:979-985.

56. Wintermark M, Reichhart M, Cuisenaire O, et al. Comparison of admission perfusion computed tomography and qualitative diffusion- and perfusion-weighted magnetic resonance imaging in acute stroke patients. Stroke 2002;33:2025-2031.

57. Hacke W, Furlan AJ, Al-Rawi Y, et al. Intravenous desmoteplase in patients with acute ischaemic stroke selected by MRI perfusiondiffusion weighted imaging or perfusion CT (DIAS-2): A prospective, randomised, double-blind, placebo-controlled study. Lancet Neurol 2009;8:141-150.

58. Marks MP, Olivot JM, Kemp S, et al. Patients with acute stroke treated with intravenous tPA 3-6 hours after stroke onset:
Correlations between MR angiography findings and perfusionand diffusion-weighted imaging in the DEFUSE study. Radiology 2008;249:614-623.

59. Davis SM, Donnan GA, Parsons MW, et al. Effects of alteplase beyond $3 \mathrm{~h}$ after stroke in the echoplanar imaging thrombolytic evaluation trial (EPITHET): A placebo-controlled randomised trial. Lancet Neurol 2008;7:299-309.

60. Wintermark M, Albers GW, Alexandrov AV, et al. Acute stroke imaging research roadmap. AJNR Am J Neuroradiol 2008;29: e23-30.

61. Provenzale JM, Wintermark M. Optimization of perfusion imaging for acute cerebral ischemia: Review of recent clinical trials and recommendations for future studies. AJR Am J Roentgenol 2008;191:1263-1270.

62. Kidwell CS, Wintermark M. The Role of CT and MRI in the Emergency Evaluation of Persons with Suspected Stroke. Curr Neurol Neurosci Rep 2010;10:21-28. 